\title{
Evaluation of Maize Genotypes against Maydis Leaf Blight Caused by Bipolaris maydis (Nisikado and Miyake) Shoemaker under Artificial Epiphytotic Conditions
}

\author{
Manjeet Singh $^{1 *}$, Rakesh Mehra ${ }^{2}$ and Vinod Kumar Malik ${ }^{1}$ \\ ${ }^{1}$ Department of Plant Pathology, ${ }^{2}$ Department of Plant Pathology, \\ CCS Haryana Agricultural University, RRS, Karnal, Haryana, India \\ *Corresponding author
}

\section{A B S T R A C T}

Keywords

Maydis leaf blight,

Maize, Bipolaris

maydis, Genotypes,

Resistance

Article Info

Accepted:

10 April 2018

Available Online:

10 May 2018

Maydis leaf blight is a serious foliar fungal disease of maize, may cause up to 41 per cent losses in grain yield. The present studies were undertaken to find out the stable sources of maydis leaf blight resistance. A set of one hundred and two maize inbred lines were evaluated against maydis leaf blight incited by Bipolaris maydis under artificial epiphytotic conditions at Regional Research Station, Karnal, CCSHAU during Kharif 2015 and 2016. The incidence of maydis leaf blight was recorded following 1-5 rating scale and the genotypes evaluated were grouped into four categories viz. resistant, moderately resistant, moderately susceptible and susceptible based on their disease reaction. Our results indicated that six genotypes (HKI 1128, HKI 5072-2BT (1-2-2), HKI 1352-58-9, MBR-139, HKI 190 and HKI 1352-58-9-2) were found resistant, thirty six genotypes were moderately resistant, fifty genotypes showed moderately susceptible reaction and ten exhibited susceptible reaction against maydis leaf blight. Thus, the identified six resistant genotypes could be utilized for developing promising maize hybrids with inbuilt resistance to maydis leaf blight disease of maize.

\section{Introduction}

Maize or corn (Zea mays L. $2 \mathrm{~N}=20$ ) is one of the most important cereal crops in the world and is grown in more than 150 countries. The major maize producing countries are USA, China, Brazil, Mexico, France and India. Maize is the third most important food grain in India next to wheat and rice and it occupies an area of 9.26 million hectares having production of 2.4 million tones with an average productivity of 2.57 t/ha (Anonymous, 2016). In Haryana, it is cultivated in 8 thousand hectares with annual production and average yield of 18,000 tones and $2250 \mathrm{~kg} / \mathrm{ha}$, respectively during Kharif (Anonymous, 2016). It is being used as feed $(63 \%)$, food $(23 \%)$, starch industries $(12 \%)$, seed and miscellaneous use $(2 \%)$ in India.

Maize is affected by as many as 112 diseases on a global basis and out of these 35 has been 
reported from India. The diseases results not only in yield reduction but also deteriorate the value and quality of the grain. Among the wide disease spectrum the more serious diseases of maize are leaf blight, downy mildew, stalk rot and rust (Kumar et al., 2013). Out of these, maydis leaf blight (MLB) is considered as one of the most serious diseases and has attained the status of the economically important disease (Malik et al., 2017).

The disease is widely distributed in India during Kharif season. In India, MLB appears in hills, plains and peninsular parts. The currently predominant form of $C$. heterostrophus is Race $\mathrm{O}$, in severity, which can cause yield losses of up to $40 \%$ (Byrnes et al., 1989). Maydis leaf blight appears every year and incidence ranging from 20-85 per cent in Haryana (Mehra et al., 2012). Host resistance is the best way for the management of the diseases. Keeping in mind the economic importance of MLB disease in Indian as well as international perspectives, there is an immediate need to identify the stable sources of resistance. Therefore, in the present study attempts were made to screen inbred lines against maydis leaf blight under artificial inoculation conditions.

\section{Materials and Methods}

In the present investigation, one hundred and two maize inbred lines of maize were evaluated at research area of Chaudhary Charan Singh Haryana Agricultural University, Regional Research Station, Karnal during Kharif 2015 and 2016 under artificial epiphytotic conditions conducive to maydis leaf blight. Karnal is considered as hotspot of maydis leaf blight disease of maize.

Two lines of each inbred consisting of $3 \mathrm{~m}$ length with a row to row and plant to plant spacing of $75 \mathrm{~cm}$ and $20 \mathrm{~cm}$, respectively.
Experiments were laid out in a randomized complete block design with three replications and recommended package of practices were followed for raising the crop (Anonymous, 2011).

The inoculation for maydis leaf blight was performed by culturing Bipolaris maydis on sorghum [Sorghum bicolor (L.)] seed following the method of Lim (1975). The plants were inoculated on 30-35 days old plants with a fine powder of these sorghum grains by putting a pinch of this powdered inoculum in the leaf whorl in late afternoon to avoid the maximum day temperature during incubation period and second inoculation was done after a week of first inoculation.

The incidence of maydis leaf blight was recorded on randomly selected ten plants in each replication following 1-5 rating scale (Payak and Sharma, 1983) as follows:

\section{Maydis leaf blight disease rating scale (1-5)}

1.0 - Very slight to slight infection, one or two to few scattered lesions on lower leaves

2.0 - Light infection, moderate number of lesions on lower leaves only

3.0 - Moderate infection, abundant lesions on lower leaves, few on middle leaves

4.0 - Heavy infections abundant lesions on lower and middle leaves, extending to upper leaves

5.0 - Very heavy infection, lesions abundant on almost all leaves; plants prematurely dry or killed by the disease.

In order to get the final disease rating of a genotype, the mean disease score of each replication was averaged and the genotypes were categorized as represented in Table 1. 


\section{Results and Discussion}

All the genotypes showed significant variation in disease reaction. Perusal of data presented in Table 2 and 3 indicated that all the genotypes showed resistant to susceptible reaction against maydis leaf blight of maize. The results revealed that the maydis leaf blight reaction during both the years Kharif 2015 and 2016 varied from 1.5 to 5.0, indicating good differentiation among genotypes. Out of 102 genotypes evaluated, six genotypes viz. HKI 1128, HKI 5072-2BT (1-2-2), HKI 1352-58-9, HKI MBR-139, HKI 190 and HKI 1352-58-92 were found resistant $(\leq 2.0)$ to MLB disease with mean disease score ranging from 1.58 to 1.92 , thirty six genotypes viz. HKI 335, HKI 766-2WG, HKI 1040-7, HKI 1015-6, HKI 1105-2MLY, HKI 1155-1-2, HKI 1155-1-1 ER (1+3), HKI LM-6, HKI LM-16, HKI 1647-2, HKI 164-7-7ER3, HKI $170(1+2)$, HKI 193-1, HKI 193-2, HKI 194-6, HKI C-141, HKI 1332, HKI 1341, HKI 1344, HKI 13474LT, HKI 1348-6-2, HKI PC-8, HKI PC-8-3, HKI 1352-2FLT (19+20), HKI 766-2VWG, HKI 1105-2MLO, HKI 288-2, HKI 295, HKI 164-7-2-1-2, HKI 191-1-2-5, HKI C-323, HKI SC-806, HKI SHAIHID 1ER-12, HKI 1115-13, HKI 72287-6 and HKI 164D-3-3 were moderately resistant (2.1-3.0) with mean disease score ranging from 2.58 to 3.00 and fifty genotypes viz. HKI 139, HKI 323, HKI 325-A, HKI 325-17A, HKI 577, HKI 766 RG, HKI 1011, HKI 1040-4, HKI 1105-6, HKI 161, HKI 163, HKI 5072-2BT (1-2)3, HKI 1347-4LT (1+2+3), HKI 1348, HKI 1352, HKI 1378, HKI PC-1, HKI PC-3, HKI PC-4, HKI PC-7, HKI PC-1414-3, HKI PC-1473-5, HKI PC-1558-4, HKI PC-5070, HKI PC72181-4, HKI 1352-2FLT $(3+11+18)$, HKI 1105, HKI L287, HKI 327 T, HKI 327 D, HKI 488, HKI 586-1D, HKI 1025, HKI 34 (1+2)-1, HKI 193-2ER-2, HKI TALLAR PF1-2, HKI 3-4-8 ER-6, HKI 325-17AN, HKI GD-8WG, HKI NP-77ER-12, HKI NP-80ER2, HYD-4005, HKI 139-1, HKI 164-3-3-2,
HKI 164-4 (1-3), HKI 766-2, HKI 1105ER, HKI 1035-11, HKI 149-1 and HKI 14$1(1+2+3)$ were found moderately susceptible (3.1-4.0) with mean disease score ranged from 3.08 to 3.92. However, ten genotypes (HKI 34-7, HKI 295 GT, HKI 488-1RG, HKI 536CBT, HKI 1040-5, HKI PCBT-3, HKI PC-4B, HKI 209, HKI 536 YN and HKI PC-4A) exhibited susceptible reaction $(\geq 4.1)$ with mean disease score range of 4.17 to 4.59 against maydis leaf blight.

In the previous studies researchers have found sources of resistance against MLB. Sharma et al., (2003) reported inbred lines CM 104 and CM 105 as resistant sources to maydis leaf blight of maize. Dhanju and Dass (2005) also evaluated a number of maize lines resistant to MLB under artificial inoculation conditions and identified multiple disease resistant inbred lines (HKI-295, HKI-1354, HKI-1348-6 and HKI-488) which are used as parents for released/identified hybrids like HHM-1, HM-5 and HM-6 resistant to maydis leaf blight. Kaur et al., (2010) also recorded genotype E 10 (LET DR 99 x Ent 49-2) as resistant and five genotypes (E1, E2, E8, E9 and E15) moderately resistant to maydis leaf blight.

Seventeen newly developed QPM inbred lines were screened against maydis leaf blight and charcol rot by Kumar et al., (2013). Inbred lines DQL 2015 and DQL 2071 were found as resistant for MLB and moderately resistant to charcoal rot. Three inbred lines (DQL 2015, DQL 2031 and DQL 2071) were found as resistant to MLB disease by Malik et al., 2015. Kumar et al., (2016) carried out inheritance study on 112 inbred maize lines and identified the stable source of MLB resistance, and MLB resistance linked markers from diverse background in the Indian adapted tropical maize genotypes. Goudar and Harlapur (2016) found only two inbred lines, BM-55 and BM-148 with high level of resistance and nine hybrids resistant. 
Table.1 The mean disease score of each replication was averaged and the genotypes were categorized as follows

\begin{tabular}{|c|l|}
\hline Score & Disease reaction \\
\hline$\leq \mathbf{2 . 0}$ & Resistant $(\mathbf{R})$ \\
\hline $\mathbf{2 . 1 - 3 . 0}$ & Moderately Resistant (MR) \\
\hline $\mathbf{3 . 1 - 4 . 0}$ & Moderately Susceptible (MS) \\
\hline $\mathbf{2 4 . 1}$ & Susceptible (S) \\
\hline
\end{tabular}

Table.2 Screening of maize inbred lines against maydis leaf blight (Bipolaris maydis) under field conditions during Kharif 2015 and 2016

\begin{tabular}{|c|c|c|c|}
\hline $\begin{array}{l}\text { Disease } \\
\text { score }\end{array}$ & Reaction & $\begin{array}{l}\text { No. of } \\
\text { inbred } \\
\text { lines }\end{array}$ & Inbred lines \\
\hline$\leq \mathbf{2 . 0}$ & Resistant (R) & 6 & $\begin{array}{l}\text { HKI 1128, HKI 5072-2BT (1-2-2), HKI 1352-58-9, } \\
\text { MBR-139, HKI } 190 \text { and HKI 1352-58-9-2 }\end{array}$ \\
\hline 2.1-3.0 & $\begin{array}{l}\text { Moderately } \\
\text { Resistant } \\
\quad(\mathrm{MR})\end{array}$ & 36 & $\begin{array}{l}\text { HKI 335, HKI 766-2WG, HKI 1040-7, HKI 1015-6, } \\
\text { HKI 1105-2MLY, HKI 1155-1-2, HKI 1155-1-1 ER } \\
\text { (1+3), LM-6, LM-16, HKI 164-7-2, HKI 164-7-7ER3, } \\
\text { HKI 170 (1+2), HKI 193-1, HKI 193-2, HKI 194-6, } \\
\text { HKI C-141, HKI 1332, HKI 1341, HKI 1344, HKI } \\
\text { 1347-4LT, HKI 1348-6-2, HKI PC-8, HKI PC-8-3, } \\
\text { HKI 1352-2FLT (19+20), HKI 766-2VWG, HKI 1105- } \\
\text { 2MLO, HKI 288-2, HKI 295, HKI 164-7-2-1-2, HKI } \\
\text { 191-1-2-5, HKI C-323, HKI SC-806, HKI SHAIHID } \\
\text { 1ER-12, HKI 1115-1-3, HKI 72287-6 and HKI 164D- } \\
3-3\end{array}$ \\
\hline $3.1-4.0$ & $\begin{array}{l}\text { Moderately } \\
\text { Susceptible } \\
\text { (MS) }\end{array}$ & 50 & $\begin{array}{l}\text { HKI 139, HKI 323, HKI 325-A, HKI 325-17A, HKI } \\
577, \text { HKI } 766 \text { RG, HKI 1011, HKI 1040-4, HKI 1105- } \\
6 \text {, HKI 161, HKI 163, HKI 5072-2BT (1-2)3, HKI } \\
\text { 1347-4LT (1+2+3), HKI 1348, HKI 1352, HKI 1378, } \\
\text { HKI PC-1, HKI PC-3, HKI PC-4, HKI PC-7, HKI PC- } \\
\text { 1414-3, HKI PC-1473-5, HKI PC1558-4, HKI PC- } \\
\text { 5070, HKI PC-72181-4, HKI 1352-2FLT (3+11+18), } \\
\text { HKI 1105, HKI L287, HKI } 327 \text { T, HKI 327 D, HKI } \\
\text { 488, HKI 586-1D, HKI 1025, HKI } 34 \text { (1+2)-1, HKI } \\
\text { 193-2ER-2, HKI TALLAR PF-1-2, HKI 3-4-8 ER-6, } \\
\text { HKI 325-17AN, HKI GD-8WG, HKI NP-77ER-12, } \\
\text { HKI NP-80ER-2, HKI HYD-4005, HKI 139-1, HKI } \\
\text { 164-3-3-2, HKI 164-4 (1-3), HKI 766-2, HKI 1105ER, } \\
\text { HKI 1035-11, HKI 149-1 and HKI 14-1(1+2+3) }\end{array}$ \\
\hline 4.1-5.0 & $\begin{array}{l}\text { Susceptible } \\
\text { (S) }\end{array}$ & 10 & $\begin{array}{l}\text { HKI 3-4-7, HKI } 295 \text { GT, HKI 488-1RG, HKI 536- } \\
\text { CBT, HKI 1040-5, HKI PCBT-3, HKI PC-4B, HKI } \\
\text { 209, HKI } 536 \text { YN and HKI PC-4A }\end{array}$ \\
\hline
\end{tabular}


Table.3 Disease rating of maize inbred lines against maydis leaf blight (Bipolaris maydis) under field conditions during Kharif 2015 and 2016

\begin{tabular}{|c|c|c|c|c|c|}
\hline Sr. No. & Inbred lines & Kharif 2015 & Kharif 2016 & Mean & Reaction \\
\hline 1 & HKI 3-4-7 & 4.17 & 4.33 & 4.25 & $\mathrm{~S}$ \\
\hline 2 & HKI 139 & 3.33 & 3.50 & 3.42 & MS \\
\hline 3 & HKI 295 GT & 4.17 & 4.33 & 4.25 & $\mathrm{~S}$ \\
\hline 4 & HKI 323 & 3.33 & 3.50 & 3.42 & MS \\
\hline 5 & HKI 325-A & 3.33 & 3.50 & 3.42 & MS \\
\hline 6 & HKI 325-17A & 3.50 & 3.83 & 3.67 & MS \\
\hline 7 & HKI 335 & 3.00 & 3.00 & 3.00 & MR \\
\hline 8 & HKI 488-1RG & 4.17 & 4.33 & 4.25 & $\mathrm{~S}$ \\
\hline 9 & HKI 536-СBT & 4.17 & 4.17 & 4.17 & $\mathrm{~S}$ \\
\hline 10 & HKI 577 & 3.33 & 3.50 & 3.42 & MS \\
\hline 11 & HKI 766 RG & 3.17 & 3.50 & 3.33 & MS \\
\hline 12 & HKI 766-2WG & 2.50 & 2.83 & 2.67 & MR \\
\hline 13 & HKI 1011 & 3.50 & 3.67 & 3.58 & MS \\
\hline 14 & HKI 1015-6 & 2.33 & 2.83 & 2.58 & MR \\
\hline 15 & HKI 1040-4 & 3.33 & 3.50 & 3.42 & MS \\
\hline 16 & HKI 1040-5 & 4.33 & 4.50 & 4.42 & $\mathrm{~S}$ \\
\hline 17 & HKI 1040-7 & 2.83 & 3.17 & 3.00 & MR \\
\hline 18 & HKI 1105-6 & 3.33 & 3.50 & 3.42 & MS \\
\hline 19 & HKI 1105-2MLY & 2.83 & 3.00 & 2.92 & MR \\
\hline 20 & HKI 1128 & 1.50 & 1.67 & 1.58 & $\mathrm{R}$ \\
\hline 21 & HKI 1332 & 2.33 & 3.50 & 2.92 & MR \\
\hline 22 & HKI 1155-1-2 & 2.83 & 3.00 & 2.92 & MR \\
\hline 23 & HKI $1155-1-1$ ER $(1+3)$ & 2.50 & 3.00 & 2.83 & MR \\
\hline 24 & HKI LM-6 & 2.50 & 3.50 & 3.00 & MR \\
\hline 25 & HKI LM-16 & 2.50 & 3.33 & 2.91 & MR \\
\hline 26 & HKI 161 & 3.50 & 3.67 & 3.58 & MS \\
\hline 27 & HKI 163 & 3.33 & 3.50 & 3.42 & MS \\
\hline 28 & HKI 164-7-2 & 2.50 & 3.00 & 2.75 & MR \\
\hline 29 & HKI 164-7-7ER3 & 2.50 & 2.67 & 2.58 & MR \\
\hline 30 & HKI $170(1+2)$ & 2.50 & 3.00 & 2.75 & MR \\
\hline 31 & HKI 193-1 & 3.00 & 3.00 & 3.00 & MR \\
\hline 32 & HKI 193-2 & 2.17 & 3.50 & 2.84 & MR \\
\hline 33 & HKI 194-6 & 2.50 & 3.00 & 2.75 & MR \\
\hline 34 & HKI 5072-2BT (1-2-2) & 1.83 & 1.83 & 1.83 & $\mathrm{R}$ \\
\hline 35 & HKI 5072-2BT (1-2)3 & 3.50 & 3.50 & 3.50 & MS \\
\hline 36 & HKI MBR-139 & 1.50 & 1.83 & 1.67 & $\mathrm{R}$ \\
\hline 37 & HKI C-141 & 2.83 & 3.00 & 2.92 & MR \\
\hline 38 & HKI 1344 & 2.33 & 3.00 & 2.67 & MR \\
\hline 39 & HKI 1347-4LT & 3.00 & 3.00 & 3.00 & MR \\
\hline 40 & HKI 1347-4LT $(1+2+3)$ & 3.33 & 3.50 & 3.42 & MS \\
\hline 41 & HKI 1348 & 3.50 & 4.00 & 3.75 & MS \\
\hline 42 & HKI 1348-6-2 & 2.50 & 3.50 & 3.00 & MR \\
\hline 43 & HKI 1352 & 3.33 & 3.50 & 3.42 & MS \\
\hline
\end{tabular}




\begin{tabular}{|c|c|c|c|c|c|}
\hline 44 & HKI 1378 & 3.33 & 3.67 & 3.50 & MS \\
\hline 45 & HKI PC-1 & 3.50 & 3.67 & 3.58 & MS \\
\hline 46 & HKI PC-3 & 3.33 & 3.50 & 3.42 & MS \\
\hline 47 & HKI PCBT-3 & 4.17 & 4.33 & 4.25 & $\mathrm{~S}$ \\
\hline 48 & HKI PC-4 & 3.33 & 3.50 & 3.42 & MS \\
\hline 49 & HKI PC-4A & 3.50 & 5.00 & 4.25 & $\mathrm{~S}$ \\
\hline 50 & HKI PC-4B & 4.17 & 5.00 & 4.59 & $\mathrm{~S}$ \\
\hline 51 & HKI PC-7 & 3.33 & 3.50 & 3.42 & MS \\
\hline 52 & HKI PC-8 & 2.83 & 3.33 & 3.08 & MR \\
\hline 53 & HKI PC-8-3 & 2.50 & 3.00 & 2.75 & MR \\
\hline 54 & HKI PC-1414-3 & 3.50 & 3.83 & 3.67 & MS \\
\hline 55 & HKI PC-1473-5 & 3.17 & 3.83 & 3.50 & MS \\
\hline 56 & HKI PC1558-4 & 3.33 & 3.50 & 3.42 & MS \\
\hline 57 & HKI PC-5070 & 3.33 & 3.50 & 3.42 & MS \\
\hline 58 & HKI PC-72181-4 & 3.50 & 4.00 & 3.75 & MS \\
\hline 59 & HKI 1352-58-9-2 & 1.67 & 2.00 & 1.83 & $\mathrm{R}$ \\
\hline 60 & HKI 1352-2FLT (19+20) & 2.50 & 3.00 & 2.75 & MR \\
\hline 61 & HKI 1352-2FLT $(3+11+18)$ & 3.33 & 3.83 & 3.58 & MS \\
\hline 62 & HKI 766WG & 2.33 & 3.00 & 2.67 & MR \\
\hline 63 & HKI 1105 & 3.33 & 4.00 & 3.67 & MS \\
\hline 64 & HKI 1105-2MLO & 3.17 & 3.83 & 3.50 & MR \\
\hline 65 & HKI 209 & 4.33 & 4.50 & 4.42 & $\mathrm{~S}$ \\
\hline 66 & HKI L287 & 3.33 & 4.00 & 3.67 & MS \\
\hline 67 & HKI 288-2 & 2.83 & 3.00 & 2.92 & MR \\
\hline 68 & HKI 295 & 2.83 & 3.17 & 3.00 & MR \\
\hline 69 & HKI $327 \mathrm{~T}$ & 3.83 & 4.00 & 3.92 & MS \\
\hline 70 & HKI 327 D & 3.83 & 4.00 & 3.92 & MS \\
\hline 71 & HKI 488 & 3.33 & 3.83 & 3.58 & MS \\
\hline 72 & HKI 536 YN & 4.17 & 4.83 & 4.50 & $\mathrm{~S}$ \\
\hline 73 & HKI 586-1D & 3.33 & 4.00 & 3.67 & MS \\
\hline 74 & HKI 1025 & 3.83 & 4.00 & 3.92 & MS \\
\hline 75 & HKI 34 (1+2)-1 & 3.33 & 4.00 & 3.67 & MS \\
\hline 76 & HKI 164-7-2-1-2 & 2.33 & 2.83 & 2.58 & MR \\
\hline 77 & HKI 190 & 1.67 & 2.00 & 1.83 & $\mathrm{R}$ \\
\hline 78 & HKI 191-1-2-5 & 2.83 & 3.00 & 2.92 & MR \\
\hline 79 & HKI 193-2ER-2 & 3.33 & 4.00 & 3.67 & MS \\
\hline 80 & HKI C-323 & 2.80 & 3.00 & 2.90 & MR \\
\hline 81 & HKI SC-806 & 3.00 & 3.00 & 3.00 & MR \\
\hline 82 & HKI TALLAR PF-1-2 & 3.33 & 3.83 & 3.58 & MS \\
\hline 83 & HKI SHAIHID 1ER-12 & 2.83 & 3.00 & 2.92 & MR \\
\hline 84 & HKI 3-4-8 ER-6 & 3.33 & 3.00 & 3.17 & MS \\
\hline 85 & HKI 325-17AN & 3.33 & 4.00 & 3.67 & MS \\
\hline 86 & HKI 1115-1-3 & 2.83 & 3.00 & 2.92 & MR \\
\hline 87 & HKI GD-8WG & 3.50 & 4.00 & 3.75 & MS \\
\hline 88 & HKI NP-77ER-12 & 3.50 & 4.00 & 3.75 & MS \\
\hline 89 & HKI NP-80ER-2 & 3.50 & 4.00 & 3.75 & MS \\
\hline 90 & HKI HYD-4005 & 3.33 & 3.83 & 3.58 & MS \\
\hline
\end{tabular}




\begin{tabular}{|c|l|l|l|l|l|}
\hline 91 & HKI 72287-6 & 2.83 & 3.00 & 2.92 & MR \\
\hline $\mathbf{9 2}$ & HKI 139-1 & 3.83 & 4.00 & 3.92 & MS \\
\hline $\mathbf{9 3}$ & HKI 164-3-3-2 & 3.50 & 4.00 & 3.75 & MS \\
\hline $\mathbf{9 4}$ & HKI 164D-3-3 & 2.83 & 3.00 & 2.92 & MR \\
\hline $\mathbf{9 5}$ & HKI 164-4 (1-3) & 3.33 & 3.00 & 3.17 & MS \\
\hline $\mathbf{9 6}$ & HKI 1341 & 2.50 & 3.50 & 3.00 & MR \\
\hline $\mathbf{9 7}$ & HKI 1352-58-9 & 1.83 & 2.00 & 1.92 & R \\
\hline $\mathbf{9 8}$ & HKI 766-2 & 3.50 & 4.00 & 3.75 & MS \\
\hline $\mathbf{9 9}$ & HKI 1105EARLY & 3.33 & 3.83 & 3.58 & MS \\
\hline $\mathbf{1 0 0}$ & HKI 1035-11 & 3.83 & 4.00 & 3.92 & MS \\
\hline $\mathbf{1 0 1}$ & HKI 149-1 & 3.50 & 4.00 & 3.75 & MS \\
\hline $\mathbf{1 0 2}$ & HKI 14-1(1+2+3) & 3.83 & 4.00 & 3.92 & MS \\
\hline
\end{tabular}

Malik et al., (2017) identified multiple disease resistant maize accessions against MLB and banded leaf and sheath blight (BLSB) after screening of 94 normal hybrids and 48 special corns genotypes. Srivastava $e t$ al., (2017) found twenty six inbreds resistant and twenty five moderately resistant to maydis leaf blight. In the present investigation keeping in mind, the resistance to maydis leaf blight, HKI 1128, HKI 5072-2BT (1-2-2), HKI 1352-58-9, HKI MBR-139, HKI 190 and HKI 1352-58-9-2 which showed resistant response, seemed to be highly promising genotype which could further be used in maize breeding programs. The further use of selected inbred will strengthens resistance breeding, developing resistant maize varieties with inbuilt resistance against maydis leaf blight and further development of management strategies for the control of maydis leaf blight of maize.

It may be concluded that among the inbred lines evaluated against maydis leaf blight, six genotypes viz. HKI 1128, HKI 5072-2BT (12-2), HKI 1352-58-9, HKI MBR-139, HKI 190 and HKI 1352-58-9-2 were found resistant. Simultaneously, thirty six genotypes including HKI 335, HKI 766-2WG, HKI 1040-7, HKI 1015-6, HKI 1105-2MLY etc were moderately resistant which can be used for further resistance breeding against the pathogen. Genotypes including HKI 3-4-7, HKI 295 GT, HKI 488-1RG exhibited susceptible reaction with mean disease score range of 4.17 to 4.59 against maydis leaf blight.

\section{Acknowledgements}

All sort of assistance rendered by Director of Research, Chaudhary Charan Singh Haryana Agricultural University, Hisar for the above study is gratefully acknowledged.

\section{References}

Anonymous, 2011. Package and practices of CCS Haryana Agricultural University, Hisar. pp. 40-49.

Anonymous, 2016. Statistical year book India 2014-15. Ministry of Statistics and Programme implementation, Govt. of India.

http://www.mospi.gov.in/downloadreports.

Byrnes, K.J., Pataky, J.K. and White, D.G. 1989. Relationships between yield of three maize hybrids and severity of southern leaf blight caused by race $\mathrm{O}$ of Bipolaris maydis. Plant Diseases, 73: 834-840.

Dhanju, K.S. and Dass, S. 2005. Identification of multiple disease resistant lines and their use in developing disease free maize hybrids. Annals of Agri-Bio Research, 10(1): 35-37. 
Goudar, S. and Harlapur, S.I. 2016. Evaluation of maize inbred lines and hybrids for resistance to maydis leaf blight. Journal of Farm Sciences, 29(3): 408-409.

Kaur, H., Hooda K.S and Khokhar, M.K. 2014. Maydis leaf blight of maize: Historical perspective, impact and present status. Maize Journal, 3(1\&2): 1-8.

Kumar, B., Hooda, K.S., Gogoi, R., Kumar, V., Kumar, S., Abhishek, A., Bhati, P., Sekhar, J.C., Yathish, K.R., Singh, V., Das, A., Mukri, G., Varghese, E., Kaur, H., Malik, V. and Yadav, O.P. 2016. Inheritance study and stable sources of maydis leaf blight (Cochliobolus heterostrophus) resistance in tropical maize germplasm. Cereal Research Communications, 44(3): 424-434.

Kumar, R., Hooda, K.S., Olakh, D.S., Kaur, H., Malik, V. and Kumar, S. 2013. Reaction of QPM inbred lines against Maydish Leaf Blight (MLB) and Charcol Rot. Electronic Journal of Plant Breeding, 4 (4): 1280-1283.

Lim, S.M., 1975. Heterotic effect of resistance in maize to Helminthosporium maydis race $\mathrm{O}$. Phytopathology, 65: 1117-1120.

Malik, V.K., Gogoi, R., Hooda, K.S. and Singh, M. 2017. Identification of multiple disease resistant maize accessions. Indian Phytopathology, 70(1): 80-85.
Malik, V.K., Kumar, R., Jaglan, M.S. and Kamboj, M. C. 2015. Identification of new sources of resistance against maydis leaf blight (MLB) and banded leaf and sheath blight (BLSB) diseases in quality protein maize. Progressive Research - An International Journal, 10(1): 606-608.

Mehra, R., Kamboj, M.C., Mehla, J.C., Lal, M. and Meharchand. 2012. Status of maize diseases and their management in Haryana. In. Proc. National sustainable Agriculture and Food security: Challenges in changing climate, March 27-28, 2012, CCS HAU, Hisar, pp 217.

Payak, M.M. and Sharma, R.C. 1983. Disease rating scales in maize in India. In: Techniques of scoring for resistance to important diseases of maize. All India Coordinated Maize Improvement Project, IARI, New Delhi, pp. 1-4.

Sharma, R.C., Rai, S.N., Mukherjee, B.K. and Gupta, N.P. 2003. Assessment potential of resistance sources for the enhancement of resistance to maydis leaf blight (Bipolaris maydis) in maize (Zea mays L.). Indian Journal of Genetics and Plant Breeding, 63(1): 3336.

Srivastava, R.P., Singh, R., Mani, V.P., Khandelwal, R.S. and Alam, M. 2017. Additional sources of resistance for southern corn leaf blight in Indian maize germplasm. Ekin Journal of Crop Breeding and Genetics, 3(1): 41-44.

\section{How to cite this article:}

Manjeet Singh, Rakesh Mehra and Vinod Kumar Malik. 2018. Evaluation of Maize Genotypes against Maydis Leaf Blight Caused by Bipolaris maydis (Nisikado and Miyake) Shoemaker under Artificial Epiphytotic Conditions. Int.J.Curr.Microbiol.App.Sci. 7(05): 1006-1013. doi: https://doi.org/10.20546/ijcmas.2018.705.125 